\title{
Coccidioidomicosis en caninos y felinos: hallazgos clínicos, diagnóstico y tratamiento
}

\author{
Canine and feline coccidioidomycosis: clinical findings, diagnosis and \\ treatment
}

\author{
Martínez Cepeda GE1* ${ }^{*}$, Revelo Ruales AP²
}

1. Docente de la Cátedra de Biología, Carrera de Medicina Veterinaria y Zootecnia, Facultad de Ciencias Agropecuarias de la Universidad Técnica de Babahoyo. Gerente General de Centro de Diagnóstico Veterinario "Santo Domingo" (CEDIVET) 2. Profesional independiente.

* Correo electrónico del autor de contacto: galomartinez88@gmail.com

\begin{abstract}
Resumen
La coccidioidomicosis es una enfermedad endémica de las zonas áridas de América. Se han identificado dos especies de este género, Coccidioides immitis y Coccidioides posadasii. La variedad de especies susceptibles, incluyendo a los humanos, hace que su diagnóstico tenga una gran relevancia. Esta enfermedad no presenta una sintomatología específica que facilite su identificación. Tanto en caninos como en felinos, se puede presentar como una enfermedad pulmonar leve o una enfermedad multisistémica diseminada que puede causar la muerte. El diagnóstico se basa en el hallazgo histopatológico de microabscesos (frescos) en muestras del tracto respiratorio u otros órganos con la respectiva identificación de esférulas que contengan endosporas. No obstante, a causa del desafío que representa su diagnóstico sobre todo en regiones donde no es endémica, es necesario recurrir a pruebas complementarias, como el cultivo y aislamiento, bajo las condiciones de bioseguridad adecuadas, y una posterior confirmación mediante pruebas moleculares.
\end{abstract}

\section{Palabras clave}

Coccidioides spp., coccidioidomicosis, diagnóstico, tratamiento

\begin{abstract}
Coccidioidomycosis is an endemic disease of the arid zones of America. Two species of this genus have been identified: Coccidioides immitis and Coccidioides posadasii. The variety of susceptible species, including humans, makes its diagnosis highly relevant. This disease does not have a specific symptomatology that allows its identification. Both in dogs and cats, it may present as a mild pulmonary disease or as a disseminated multisystemic disease that can cause death. The diagnosis is based on the histopathological findings of microabscesses (fresh) in respiratory or other organs with identification of spherules containing endospores. However, because of the challenge of its diagnosis, especially in regions where it is not endemic, it is necessary to proceed with complementary tests such as culture and isolation, under the appropriate biosecurity conditions, and subsequent confirmation by molecular tests.
\end{abstract}

\section{Key words}

Coccidioides spp., coccidioidomycosis, diagnosis, treatment

Fecha de recepción: 06/01/2018

Fecha de aprobación: 21/03/2018
ANALECTA Vet 2018; 38(1): 33-44

Impresa ISSN 03655 14-8 Electrónica ISSN 1514-2590

doi.org/10.24215/15142590eo23 


\section{Introducción}

La coccidioidomicosis, también llamada fiebre del valle, es una enfermedad producida por dos especies de hongos dimórficos: Coccidioides immitis (endémico del área de San Joaquín, California, EE.UU.) y Coccidioides posadasii (endémico en las restantes áreas del continente americano). La micosis es endémica en las zonas áridas del continente americano (Mehrkens et al., 2016). Estos hongos son termodimórficos, su fase micelial desarrolla a $25^{\circ} \mathrm{C}$ en el ambiente, donde producen artroconidios que son los que ingresan al hospedero susceptible y producen la infección. En el hombre y en los animales desarrolla la fase parasítica que se caracteriza por esférulas con endosporas.

Los casos clínicos son relativamente comunes en perros, llamas, primates y muchas especies no nativas mantenidas en zoológicos en las regiones endémicas. La enfermedad también fue documentada en gatos y en caballos. Los animales pueden actuar como centinelas de la enfermedad en humanos, debido a que comparten una ambiente común y a la estrecha relación existente, principalmente con los caninos domésticos (Mehrkens et al., 2016). Si bien hay varias técnicas disponibles en la actualidad para el diagnóstico de enfermedades micóticas sistémicas, el estudio histopatológico es la prueba de elección cuando es posible disponer de tejidos (RamírezRomero et al., 2016).

\section{Historia de la enfermedad}

La enfermedad fue descrita por primera vez en Buenos Aires en 1892, aunque la naturaleza fúngica de la misma fue revelada en California (Negroni, 2011).

El Dr. Bengolea, médico dermatólogo del Hospital Rawson de Buenos Aires, solicitó al estudiante de medicina Alejandro Posadas revisar una biopsia cutánea procedente de una lesión de aspecto verrugoso con diagnóstico presuntivo de "micosis fungoide". El paciente era un soldado de caballería llamado Domingo Ezcurra, nacido en la Provincia de San Luis, en 1852. Se supuso que el paciente había contraído la infección cuando prestaba servicio militar en el año de 1888 en la frontera del Chaco. Un año más tarde aparecieron las primeras manifestaciones de la enfermedad, de evolución crónica y progresiva. El Dr. Posadas observó, en el examen clínico, pápulas pruriginosas, de aspecto verrugoso, localizadas en la cabeza, espalda, abdomen y en los miembros. También observó adenopatías, algunas con reblandecimiento y escrofuloderma, mientras que otras habían bloqueado la circulación linfática ocasionando linfedema (Negroni \& Arechavala, 2006; Negroni, 2011).

Posadas y su maestro, el Dr. Wernicke, observaron el agente etiológico en los estudios histológicos, en los que observaron un proceso crónico inflamatorio, con formación de granulomas epitelioides con células gigantes, similar al tuberculoso, pero que contenían formaciones quísticas muy parecidas a las producidas por los protozoarios del género Coccidia. Como observaron que no era idéntico al género mencionado, lo clasificaron dentro del género Psorosperma, por lo que la enfermedad se llamó inicialmente psorospermiasis infectante generalizada (Canteros et al., 2009; Negroni, 2011).

Los médicos realizaron tratamientos e intervenciones quirúrgicas sin resultados favorables y la enfermedad progresó ocasionando la muerte del paciente en 1898. Se le practicó una autopsia, haciéndose evidentes lesiones en la piel, linfonódulos, pulmones, hígado y bazo. Los granulomas producidos por este agente eran similares al folículo de Koster de la tuberculosis, con necrosis caseosa en el centro, una zona media de células gigantes multinucleadas y células epitelioides y un manto de células mononucleares linfocitarias en la periferia. La única diferencia era la presencia de quistes de "psorospermias" ubicados en la zona caseosa y en el interior de las células gigantes.

Posadas inoculó macerados del material de biopsia por vía subcutánea, en la zona abdominal, en varias especies de animales. Las ranas y tortugas demostraron ser resistentes al hongo. En los monos, la infección experimental demostró ser muy grave y les causó la muerte 18 a 31 días posinoculación, mientras que en las ratas y perros la enfermedad fue más parecida a la desarrollada en el humano, crónica y ulcerativa (Negroni, 2011).

\section{Agente etiológico}

Coccidioides spp. presenta diferentes formas en su fase saprófita y parasitaria y se distingue de otros patógenos fúngicos por las características morfológicas únicas de su crecimiento en el tejido del hospedero.

\section{Posición taxonómica \\ División: Ascomycota \\ Clase: Euascomycetes \\ Orden: Onygenales \\ Familia: Onygenaceae \\ Género: Coccidioides \\ Especies: C. immitis y C. posadasii}

Hasta 2002 solo se reconocía una especie del género: $C$. immitis. Con la utilización de herramientas moleculares se propuso una nueva especie, C. posadasii, para los aislamientos de Arizona, Texas, México, Venezuela, Argentina y Brasil, reservando el nombre $C$. immitis para los aislamientos de California (Fisher et al., 2002).

Las especies de Coccidioides se identifican sobre la base del polimorfismo de cinco proteínas conservadas, además de características fenotípicas como el crecimiento en medios con alta 
concentración de sales (Castañón-Olivares et al., 2007; Gómez \& Guida, 2010; Welsh et al., 2012).

Las especies de Coccidioides habitan en zonas geográficas semiáridas, donde la temperatura anual promedio es de $30^{\circ} \mathrm{C}$, con veranos extremadamente calurosos y terrenos alcalinos arenosos o arcillosos. El hongo crece desde la superficie y hasta una profundidad de $30 \mathrm{~cm}$; los micelios permanecen latentes bajo la superficie del suelo. Tras periodos de lluvias, el agente se multiplica y libera gran número de artroconidios y su dispersión se asocia con eventos de remoción de tierra por causas naturales como el viento o por intervención humana en agricultura y construcciones. Los artroconidios son las formas infectantes que ingresan en humanos y animales por vía inhalatoria (Carrasco-Zuber et al., 2016) y, en aproximadamente 48 horas, se redondean hasta formar esférulas en el hospedero. Las esférulas maduras miden entre 20 y $150 \mu \mathrm{m}$ de diámetro y dentro de ellas se forman endosporas de 2 a $4 \mu \mathrm{m}$ de diámetro. Las esférulas maduras se rompen y liberan las endosporas, las que originan nuevas esférulas propagando la infección (Castañón-Olivares et al., 2007; Laniado-Laborin et al., 2012).

\section{Factores de virulencia}

Los artroconidios de Coccidioides spp. pueden evadir al sistema inmunológico del hospedero. La pared del conidio, derivada de la pared de la hifa original, es hidrofóbica y está adaptada para propagarse a través del aire. Esta pared constituye una capa de protección pasiva contra los mecanismos celulares de defensa, contribuyendo a la supervivencia del patógeno in situ. La elevada cantidad de endosporas liberadas por cada esférula es también un factor de virulencia. Las esférulas están recubiertas con una sustancia mucilaginosa, que actúa como agente protector contra el sistema fagocítico del hospedero. Otro factor de virulencia es la formación de granulomas, ya que el sistema inmunitario del hospedero no puede eliminar al agente causante de la enfermedad dentro del mismo (Castañón-Olivares et al., 2007; Welsh et al., 2012).

\section{Respuesta inmune}

En la coccidioidomicosis, la inmunidad mediada por células es importante para establecer una respuesta eficaz a la infección. El curso clínico puede ser, en gran medida, influenciado por la posibilidad de que la respuesta inmune sea predominantemente dirigida hacia un patrón Th1 o hacia un patrón Th2. El IFNy, citoquina asociada a Th1, activa a los macrófagos, que son posteriormente capaces de inhibir o destruir los microorganismos. En contraste, las citoquinas asociadas a Th2 promueven la producción de anticuerpos y disminuyen la regulación de las funciones de Th1.
Los anticuerpos parecen no conferir protección contra los microorganismos (Martínez-Méndez \& Semprún-Hernández, 2015). En infecciones autolimitadas, los pacientes suelen presentar un fuerte retraso en las respuestas de hipersensibilidad a antígenos coccidioidales y tienen títulos de anticuerpos bajos. Por el contrario, los pacientes con infección diseminada manifiestan típicamente un débil o nulo retraso en la respuesta de hipersensibilidad y tienen altos títulos de anticuerpos (Martínez-Méndez \& Semprún-Hernández, 2015).

Tras la recuperación de las autoinfecciones limitadas, los pacientes suelen tener inmunidad de por vida. Además de los mecanismos mediados por células $\mathrm{T}$ de la inmunidad adquirida, las células natural killer (NK) pueden jugar un papel en la resistencia innata a los organismos (DiCaudo, 2006). Mediante técnicas de inmunohistoquímica se demostró el predominio de linfocitos $\mathrm{T}$ en el centro y la periferia de los granulomas, mientras que los linfocitos B se encontraban distribuidos en la periferia de los mismos, independientemente del tejido donde se desarrolló la lesión, de la cantidad de lesiones y de hongos y de la naturaleza y gravedad de la respuesta inflamatoria (Shubitz et al., 2011).

\section{Aspectos clínicos}

La principal forma de contagio de la enfermedad es por la inhalación de los artroconidios dispersos en el ambiente. Otra forma de contagio, menos frecuente, es la inoculación directa de esporas en el tejido del animal, lo que origina la formación de un granuloma localizado (Shubitz \& Dial, 2005; Espinoza et al., 2014).

\section{Signos clínicos}

Las infecciones en los animales varían desde asintomáticas a graves y mortales. El hongo es capaz de invadir casi cualquier tejido del cuerpo. Los sitios más comunes de propagación varían con las especies, pero casi todos los tejidos y órganos pueden ser afectados. La afección del corazón o del pericardio puede ser más común en animales que en humanos. La propagación puede o no estar acompañada de signos de enfermedad sistémica (Shubitz \& Dial, 2005; Shubitz, 2007).

La diseminación hematógena y linfática de endosporas origina la enfermedad diseminada. Uno de los órganos mayormente afectados son los ojos, causando uveítis granulomatosa unilateral o bilateral; también afecta frecuentemente el ángulo de filtración, cuerpo ciliar, retina y coroides. En algunos de los casos se puede presentar hipema y hemorragia vítrea (Schaer, 2006). Los órganos que se encuentran afectados principalmente son linfonódulos, pulmón, hueso, corazón, mediastino y piel (Shubitz et al., 2011; Ortiz et al., 2015). 
Formas clínicas

La infección por Coccidioides spp. puede ser asintomática o sintomática. La primera se resuelve completamente, estableciéndose inmunidad ante una reinfección. La infección sintomática puede presentar un curso subagudo o crónico progresivo que, sin un tratamiento adecuado, puede llevar a la muerte (Shubitz \& Dial, 2005).

\section{Formas de presentación}

\section{Enfermedad pulmonar leve:}

Cursa con manifestaciones clínicas leves a inaparentes después de una a cuatro semanas de exposición. Un hospedero con el sistema inmunológico competente podrá combatir la infección e inclusive resolverla.

\section{Enfermedad multisistémica diseminada:}

Se presenta después de meses o años de la exposición y es más frecuente en perros que en gatos. Los felinos son naturalmente más resistentes a este tipo de presentación. A partir de los pulmones se disemina hacia todos los órganos por medio de las circulaciones linfática y sanguínea.

\section{Manifestaciones clínicas en caninos}

Los perros machos, jóvenes, de razas boxer, pointer, pastor australiano, beagle, scottish terrier, doberman pinscher y cocker spaniel que viven en los exteriores de las viviendas en áreas endémicas son afectados con más frecuencia (Morgan et al., 2004). El hábito del perro de olfatear aumenta las probabilidades de contraer la enfermedad (Ettinger \& Feldman, 2004; Morgan et al., 2004). En los perros y en otras especies animales se comprobó que se necesitan 10 artroconidios para producir la enfermedad clínica (Calderón-Garcidueñasa et al., 2013). La presentación de signos respiratorios se debe a la respuesta inflamatoria originada en el hospedero a causa de las endosporas. Una enfermedad respiratoria leve o subclínica se debe a una buena respuesta inmunitaria, mientras que la enfermedad pulmonar grave y diseminada se da en animales con una pobre respuesta inmunológica (Morgan et al., 2004).

En perros, el periodo de incubación puede variar entre 1 y 3 semanas. Esto se debe a la respuesta inmunitaria de cada animal, lo que también determina la severidad de la enfermedad clínica (Ettinger \& Feldman, 2004). La mayoría de los perros infectados presenta fiebre, inapetencia y pérdida de peso progresiva. También es común observar complicaciones en el aparato respiratorio con síntomas leves o, en algunos casos, hasta inaparente. La tos es el síntoma predominante. Las lesiones cutáneas con drenaje, queratitis, uveítis, ceguera aguda, cojeras asociadas con dolor de huesos largos e inflamación del periostio son otras manifestaciones que pueden observarse (Schaer, 2006; Shubitz, 2007).

Aproximadamente el $20 \%$ de los perros presenta lesiones cutáneas: nódulos, abscesos, úlceras y trayectos fistulosos. Comúnmente, las lesiones de piel son consecuencia de las infecciones óseas subyacentes, mientras las infecciones primariamente cutáneas son consecuencia de la inoculación directa del agente en la piel (Ettinger \& Feldman, 2004; Morgan et al., 2004).

A diferencia de los humanos, en los que Coccidioides spp. puede producir meningitis, en perros se producen lesiones granulomatosas en el SNC con convulsiones. Si la enfermedad involucra el corazón y el pericardio puede causar disfunción cardiaca, arritmias, síncope o muerte súbita (Shubitz, 2007).

\section{Manifestaciones clínicas en felinos}

La coccidioidomicosis no tiene predilección de sexo o raza en los gatos. Se diagnostica con mucha menos frecuencia que en los perros, por lo que la información existente es limitada (Lloret et al., 2013). Las lesiones cutáneas parecen ser el signo presente más frecuente; en general, al momento del diagnóstico la enfermedad está diseminada y es de curso grave. Pueden observarse dermatitis con lesiones que no tienden a cicatrizar, úlceras o abscesos crónicos que no responden a los antibióticos. De estas lesiones pueden obtenerse biopsias, aspirados o materiales para cultivo (Morgan et al., 2004; Shubitz, 2007). Puede producirse una linfadenopatía regional. Frecuentemente se observan signos no específicos, tales como fiebre, letargo, pérdida de peso y anorexia (Castañón-Olivares et al., 2007; Welsh et al., 2012).

En algunos gatos, la pérdida de peso severa puede ser el único signo (Gómez \& Guida, 2010). Los signos respiratorios no son tan comunes en los gatos como en los perros; sin embargo, pueden existir lesiones pulmonares sin manifestaciones clínicas. Se comprobó que el $25 \%$ de todos los gatos afectados por el hongo tienen dificultad respiratoria, pero la tos no es común. Otros sitios de diseminación son similares a los de los perros, a pesar de que los huesos no parecen estar involucrados con tanta frecuencia como en los caninos (Ettinger \& Feldman, 2004).

Los signos de afección del SNC son más variables en los gatos y pueden incluir incoordinación, convulsiones, hiperestesia y cambios de comportamiento. También pueden observarse uveítis anterior, granulomas fúngicos y desprendimiento de retina. En algunos gatos con enfermedad ocular se ha registrado inflamación periocular y masas inflamatorias en la conjuntiva palpebral (Ettinger \& Feldman, 2004; Schaer, 2006; Jinks et al., 2016). 


\section{Diagnóstico diferencial}

Todos los signos clínicos referidos son inespecíficos, por lo que son necesarias pruebas de laboratorio para determinar la causa de la enfermedad. Los diagnósticos diferenciales incluyen: tuberculosis, neoplasias, otras micosis sistémicas y neumonías de origen bacteriano. La coccidioidomicosis es, de hecho, la "gran imitadora" (DiCaudo, 20063; Castañón-Olivares et al., 2007).

\section{Métodos de diagnóstico}

El diagnóstico requiere de un conjunto de pruebas directas e indirectas, acompañadas de una anamnesis pertinente y un examen físico minucioso. En los animales con sospechas de granulomas cerebrales se debe recurrir a estudios avanzados de imágenes, tales como la resonancia magnética o la tomografía axial computarizada (Ettinger \& Feldman, 2004).

La coccidioidomicosis se diagnostica mediante la observación de Coccidioides spp. en muestras procedentes de tejidos, exudados, líquido de lavado transtraqueal o broncoalveolar, linfonódulos y líquido pleural. En el hospedero el agente forma esférulas de un diámetro de 10 a 70 $\mu \mathrm{m}$, pero se sabe que algunas pueden medir hasta $200 \mu \mathrm{m}$ (Shubitz \& Dial, 2005). En casos raros el hongo forma hifas en los tejidos o los alvéolos pulmonares (Welsh et al., 2012).

La determinación de anticuerpos en animales que padecen la enfermedad puede ser negativa o registrar valores muy bajos. Los estudios serológicos en felinos son discutidos; no obstante, se puede detectar IgM específica en un $82 \%$ de los gatos infectados e IgG específica en el 100 \% (Ettinger \& Feldman, 2004).

Los hallazgos hematológicos incluyen anemia leve, normocítica, normocrómica y no regenerativa. A menudo se aprecian neutrofilia moderada, por lo general con desviación a la izquierda, y monocitosis, mientras que la hipoalbuminemia y la hiperglobulinemia son anomalías constantes. Los hallazgos bioquímicos incluyen el incremento de las concentraciones séricas de ALT, AST, fosfatasa alcalina (FA) y bilirrubina total (BT), lo que indica compromiso hepático. La azoemia y la hiperfosfatemia pueden ser indicativas de compromiso renal.

En algunos casos se observa hipercalcemia (Morgan et al., 2004). Un estudio retrospectivo evidenció glomerulonefritis mediada por complejos inmunes en caninos y se sugirió que la coccidioidomicosis pulmonar persistente puede estimular una respuesta inmune, que puede producir daño glomerular (Mehrkens et al., 2016).

En las radiografías de tórax de caninos se suelen observar anomalías como patrones intersticiales o peribronquiolares difusos y rara vez infiltrados alveolares. Algunos autores documentaron linfadenopatía hiliar en cerca del $80 \%$ de los perros y afectación pleural manifestada por engrosamiento o derrame en menos del $65 \%$ (Ettinger \& Feldman, 2004).

\section{Métodos directos}

$\underline{\text { Examen directo en fresco }}$

En el laboratorio, el examen en fresco con $\mathrm{KOH}$ forma parte de la rutina para la observación de hongos en materiales biológicos. Con esta técnica se puede ver la doble pared. La fase tisular del microorganismo generalmente se observa como cuerpos esféricos de 10 a $70 \mu \mathrm{m}$, de pared gruesa y sin yemas, en cuyo interior presentan gran cantidad de endosporas de 2 a $5 \mu \mathrm{m}$ (esférulas inmaduras). Las esférulas más pequeñas pueden carecer de endosporas (Canteros \& SuárezÁlvarez, 2012). Las esférulas inmaduras pueden confundirse con otros elementos, por lo que deben buscarse las esférulas maduras llenas de endosporas (Ajithdoss et al., 2011).

\section{$\underline{\text { Cultivo }}$}

El desarrollo del hongo en cultivo confirma el diagnóstico. No obstante, debido a la naturaleza altamente infectante de los artroconidios, este análisis queda reservado para laboratorios de referencia con niveles de bioseguridad adecuados (laboratorio de bioseguridad nivel 3) (Schaer, 2006).

La identificación depende del hallazgo de los artroconidios característicos de la fase saprofítica de Coccidioides (Figura 1). Los artroconidios se consideran altamente infectantes y patógenos, por lo que en muchos casos se obvia el cultivo y se remiten muestras para histopatología o serología. Para la identificación definitiva se requiere la producción de esférulas en animales infectados en forma experimental o en cultivo líquido en medio de Converse a $37^{\circ} \mathrm{C}$ en atmósfera de $\mathrm{CO}_{2}$ (Ajithdoss et al., 2011; Welsh et al., 2012).

$C$. immitis y $C$. posadasii crecen en una gran variedad de medios de cultivo con antibióticos o sin ellos a $28{ }^{\circ} \mathrm{C}$ y a $37{ }^{\circ} \mathrm{C}$. En algunos casos, su desarrollo puede tardar hasta 3 semanas (Morgan et al., 2004; Welsh et al., 2012). Existen medios de cultivo como el agar Mycosel $\mathbb{R}$ o Mycobiotic $\AA$ (agar dextrosa Sabouraud con cicloheximida), en los que, entre 25 y $30{ }^{\circ} \mathrm{C}$, se observan que, macroscópicamente, al tercer día la colonia es glabra, después vellosa y luego francamente algodonosa, de color blanco grisáceo o amarillento. Microscópicamente, se observan hifas delgadas y septadas con artrosporas rectangulares de $2 \times 3$ ó $4 \times 6 \mu \mathrm{m}$. Los artroconidios, estructuras de pared gruesa, alternos a lo largo de la hifa y separados unos de otros por células disyuntoras, empiezan a aparecer entre el décimo y décimo cuarto día. El cultivo se debe realizar en 
tubo y examinar entre siete a diez días posinóculo. Para la observación microscópica, en caso de no poseer un laboratorio de nivel BSL3, es necesario colocar gotas de formol dentro del tubo antes de tomar la alícuota (DiCaudo, 2006; CastañónOlivares et al., 2007).

Conviene recordar que Coccidioides spp. en el cultivo, tanto a $36-37^{\circ} \mathrm{C}$ como a $25-30^{\circ} \mathrm{C}$, crece en forma de micelio $y$ es altamente infectante.

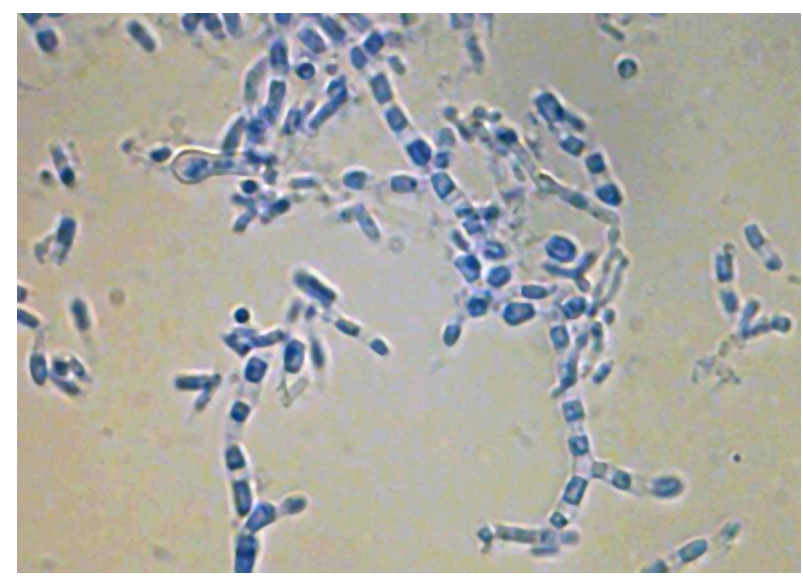

Figura 1. Fase saprófita de C. posadasii (objetivo 40X). Impronta de cultivo micológico. Tinción azul de algodón. Gentileza del Dr. Gustavo Giusiano.

\section{Histopatología}

Los pulmones son los órganos más afectados, pero la infección puede involucrar a otros órganos como los linfonódulos, piel, bazo, hígado, riñones, huesos, articulaciones y órganos del SNC. En el examen histológico se observa el granuloma coccidioidal. La presencia de necrosis con abundantes leucocitos polimorfonucleares neutrófilos y eosinófilos es una característica común en estas lesiones, pero también pueden estar presentes en otras formas clínicas. Se observan, además, linfocitos, células epitelioides, células gigantes multinucleadas y fibrosis (Welsh et al., 2012; Carrada-Bravo, 2014).

Las lesiones macroscópicas reflejan los cambios histopatológicos, que pueden incluir respuesta piogranulomatosa con neutrófilos, eosinófilos, macrófagos, células gigantes multinucleadas con células epitelioides, necrosis caseosa e hialinización o fibrosis con linfocitos en la periferia. Los cambios granulomatosos, fibrosis y ocasionalmente calcificación pueden representar lesiones crónicas, pero únicamente la presencia de Coccidioides spp. los distingue de lesiones causadas por otras enfermedades (CastañónOlivares et al., 2007).

En algunos casos se han descrito vasculitis con inflamación perivascular y endarteritis obliterante en asociación con meningitis/encefalitis coccidioidal. La reacción supurativa puede ser extensa, pero los microabscesos suelen encontrarse adyacentes a los granulomas. Ocasionalmente, la superficie de esférulas maduras teñidas con H\&E muestra un depósito eosinofílico similar al fenómeno de Splendore-Hoeppli asociado con otros hongos. Además de las tinciones con H\&E y Papanicolau, las tinciones que ponen de manifiesto polisacáridos, como la reacción de PAS y las técnicas de plata metenamina (PMN), son útiles para la identificación de Coccidioides (Vale-Echeto et al., 1999). Es posible encontrar esférulas en los tejidos y, en raras ocasiones, hifas (CastañónOlivares et al., 2007). Las esférulas miden de 10 a $70 \mu \mathrm{m}$, tienen pared gruesa y citoplasma eosinófílico. Pueden tener aspecto de cuerpo asteroide (Splendore-Hoeppli) y en lesiones no activas se deforman y adoptan formas variadas; si se rompen dan salida a las endosporas mononucleadas de 2 a $5 \mu \mathrm{m}$ de diámetro (Shubitz \& Dial, 2005).

Con PMN se observan esférulas de color parduzco a negro, mientras que el resto del tejido se tiñe de verde. Las paredes de las células fúngicas son gruesas y en el interior se pueden ver las endosporas. También se pueden ver esférulas inmaduras y endosporas libres (Canteros \& SuárezÁlvarez, 2012). La observación de formas hifales/ miceliales requiere obligatoriamente de cultivo (Castañón-Olivares et al., 2007).

Con PAS se observan esférulas de 10 a 70 $\mu \mathrm{m}$ de color rojo, mientras que el resto del tejido se tiñe de azul-violáceo. También resaltan con esta tinción las paredes que, al igual que con la PMN, se ven gruesas y en el interior pueden verse las endosporas (Canteros \& Suárez-Álvarez, 2012). Estas tinciones especiales se utilizan en caso de duda o cuando las esférulas son escasas. Cuando las esférulas son jóvenes y pequeñas, se requiere el diagnóstico diferencial con otros organismos fúngicos, tales como Cryptococcus neoformans y Blastomyces dermatitidis y, cuando las esférulas son muy grandes, con Rhinosporidium seeberi, el agente de la rinosporidiosis (Welsh et al., 2012).

Se pueden realizar técnicas de inmunohistoquímica (Figura 2), sensibles y específicas, en las que se utilizan anticuerpos policlonales específicos contra el agente, producidos en otra especie distinta de la del estudio. Luego se agrega un anticuerpo anti-especie, lo que amplifica la señal en el momento de observarla microscópicmente, previo revelado.

\section{Técnicas moleculares}

Las técnicas moleculares, tales como hibridación in situ (HIS) y PCR, pueden ayudar en la identificación de $C$. immitis / C. posadasii en tejidos embebidos en parafina. La HIS es menos sensible pero más específica que las tinciones histológicas convencionales, como la PMN. En los casos en que los microorganismos observados no puedan diferenciarse morfológicamente de otros, 
la HIS puede proporcionar confirmación específica en las secciones histológicas dificultosas. Esta técnica puede ser particularmente útil para diferenciar esférulas pequeñas e inmaduras de $C$. immitis / posadasii de Blastomyces dermatitidis y Cryptococcus neoformans (DiCaudo, 2006).

Con la técnica de RFLP se inició la caracterización de Coccidioides spp., encontrando diferencias genómicas de los aislados de distintas regiones (Zimmermann \& Snedker 1994). Por otra parte, en estudios similares se halló una especie de Coccidioides endémica de California y otra no perteneciente a esta región (Burt et al., 1997; Koufopanou et al., 1997).

Otras técnicas moleculares disponibles para la identificación de especies de Coccidioides, a partir de muestras clínicas, son la PCR anidada y la PCR en tiempo real que amplifican un fragmento del gen que codifica un antígeno específico denominado 2/antígeno rico en prolina (Ag2/PRA) (Peng et al., 1999). La primera técnica amplifica un fragmento de $340 \mathrm{pb}$ perteneciente a una parte del gen que codifica el Ag2/PRA. La secuenciación del fragmento del gen Ag2PRA amplificado por la PCR anidada permite, además, la identificación a nivel de especie de estos hongos.

La identificación mediante el uso de polimorfismos sencillos de nucleótidos (SNP) por medio de sondas taqman, es otro método de identificación de especies de Coccidioides; el mismo amplifica tres secuencias blanco: prolina 157, prolina 174 y glucosa sintetasa 192 (CastañónOlivares et al., 2007).

Debido al gran riesgo que conlleva el cultivo del hongo y la necesidad de un diagnóstico rápido y certero para la instauración de un tratamiento médico, se desarrolló una PCR en tiempo real para la identificación del género Coccidioides basada en la identificación de un fragmento de $170 \mathrm{pb}$ de la región ITS2 (Binnicker et al., 2007). La técnica demostró $100 \%$ de sensibilidad y $98,4 \%$ de especificidad en muestras de tracto respiratorio y $92,9 \%$ de sensibilidad y 98,1 \% de especificidad en muestras de tejido fresco, mientras que, en muestras tomadas de tejidos parafinados destinados a técnicas histopatológicas, se evidenció una sensibilidad del 73,4 \% y una especificidad del $100 \%$ (Binnicker et al., 2007).

Para identificar de una forma más sencilla las especies a partir de cultivos, se trabajó sobre el fragmento genómico denominado Coig de la región ITS2, el cual produce una banda de $634 \mathrm{pb}$ para C. posadasii y una banda de 720 pb para $C$. immitis (Umeyama et al., 2006).

Posteriormente se determinó que primers dirigidos a la amplificación de fragmentos de la región ITS3 - ITS4 amplifican también la región ITS2; de esta forma, se descartó el método basado en el segmento ITS2 para la diferenciación de especies del género Coccidioides (Tintelnot et al., 2007).

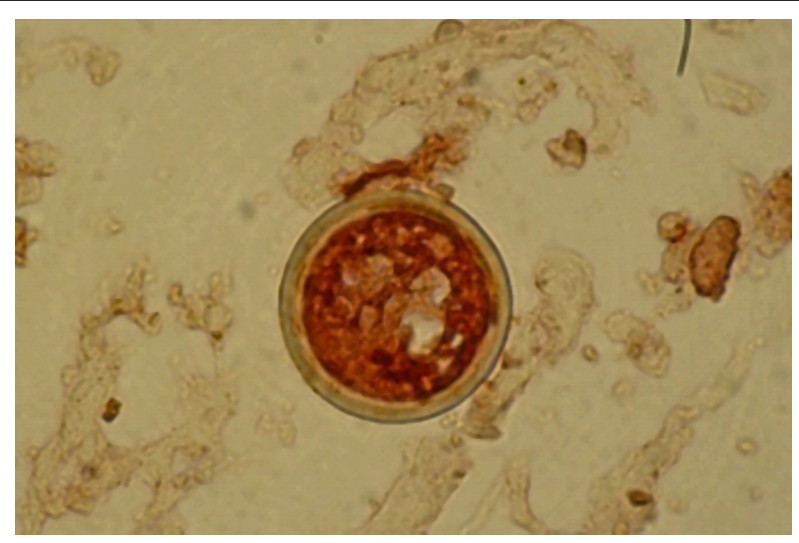

Figura 2. Endosporas de Coccidioides posadasii. Técnica inmunohistoquímica con peroxidasa de rábano picante (HRP), realizada en cortes histológicos de una biopsia de piel de perro, utilizando un anticuerpo anti-Coccidioides y diaminobenzidina como cromógeno. Aumento 1000X. Gentileza del Dr. Roberto Suárez-Álvarez. Departamento de Micología. ANLIS-INEI “Dr. Carlos G. Malbrán”.

\section{Métodos indirectos}

\section{Inmunodiagnóstico}

Prueba cutánea con coccidioidina o esferulina:

Se la realiza mediante la inyección intradérmica de $0,1 \mathrm{ml}$ de coccidioidina diluida. $\mathrm{La}$ reacción de hipersensibilidad se lee a las 24 y 48 horas y se considera positiva con una inflamación de $5 \mathrm{~mm}$ o más, con respecto a la medida preinoculación. Esta prueba se emplea en estudios epidemiológicos para determinar la prevalencia de exposición y la presencia de brotes. El uso de la coccidioidina para diagnosticar enfermedad activa en zonas endémicas ha sido descartado, ya que solo evidencia contacto con el hongo (primoinfección) y no enfermedad activa. La prueba es de valor diagnóstico solo en animales con antecedentes de reacción negativa. Una prueba cutánea negativa en un animal que antes había resultado positiva, indica anergia acompañada de enfermedad diseminada avanzada (Welsh et al., 2012).

Prueba de precipitación en tubo:

Se usa para detectar la enfermedad activa en estadios tempranos. La prueba detecta anticuerpos precipitantes IgM (Laniado-Laborin et al., 2012).

Prueba de fijación del complemento (FC):

Es una prueba que conlleva altos estándares, tanto en la preparación de reactivos como en la capacitación del personal. Es útil para el diagnóstico de la enfermedad, pero el punto clave es la importancia de la prueba para el pronóstico, mediante el seguimiento de la conversión de títulos; los anticuerpos fijadores del complemento se elevan en la enfermedad activa y diseminada. La FC es una prueba no rutinaria y la realizan 
solamente laboratorios de referencia (Ettinger \& Feldman, 2004; Morgan et al., 2004).

Inmunodifusión en gel de agar y contrainmunoelectroforesis:

Son los métodos mayormente empleados para el diagnóstico de coccidioidomicosis en la rutina diaria de laboratorios de diagnóstico de zonas endémicas (Ettinger \& Feldman, 2004). Esta prueba tiene valor diagnóstico. Arrojan resultados positivos casi al mismo tiempo que la prueba de fijación de complemento. La inmunodifusión (ID) se lleva a cabo usando una muestra de suero y antígeno de Coccidioides spp. Es la prueba más común para el diagnóstico de la enfermedad, es de fácil realización y existen reactivos comerciales. No es de elección en lugares endémicos donde el paciente presente signos escasos e inespecíficos.

Aglutinación de partículas de látex:

Se usan partículas de látex sensibilizadas con antígeno coccidioidina. Es útil para el diagnóstico de animales con signos clínicos (Ettinger \& Feldman, 2004; Morgan et al., 2004).

Otros métodos serológicos son las técnicas de anticuerpos fluorescentes específicos para descubrir esférulas en los tejidos y artroconidios en el suelo. También se han usado pruebas de inhibición de anticuerpos fluorescentes. Recientemente se probó un inmunoensayo enzimático en el cual los anticuerpos IgG son reconocidos por la proteína A/G como herramienta para estudiar la expansión de la región endémica para la coccidioidomicosis. Este ensayo, con un punto de corte adecuado, puede ser adaptado a otras especies (Chow et al., 2017).

\section{Tratamiento}

Los fármacos utilizados preferentemente son el ketoconazol, a dosis de 5 -15 $\mathrm{mg} / \mathrm{kg}$ por vía oral cada 12 horas, o el itraconazol, a dosis de 5-10 $\mathrm{mg} / \mathrm{kg}$ por vía oral cada 24 horas. El tratamiento con ketoconazol dura aproximadamente 2 meses o hasta la remisión de los signos clínicos. Una de sus desventajas es la hepatotoxicidad, presente en tratamientos prolongados (12 meses). Además, es teratogénico, limitando su uso en hembras gestantes o en hembras destinadas a la cría comercial. En perros con tratamientos de más de 12 meses puede causar cataratas (Ettinger \& Feldman, 2004; Morgan et al., 2004; Schaer, 2006; Gómez \& Guida, 2010). El itraconazol es un triazol usado para el tratamiento de la coccidioidomicosis. Es más eficaz y tiene menos efectos secundarios que el ketoconazol. El itraconazol parece tener un mejor efecto en lesiones cutáneas, subcutáneas y óseas. Su biodisponibilidad, después de la administración de la solución oral en condiciones de ayuno, es $60 \%$ mayor en comparación con cápsulas que se toman después de las comidas. El hidroxiitraconazol es un metabolito antifúngico activo de este fármaco, se acumula el doble de la tasa del fármaco original y aumenta la biodisponibilidad de itraconazol hasta un 80 \% (Welsh et al., 2012).

Los nuevos triazoles, el posaconazol y el voriconazol, son agentes antimicóticos utilizados en el tratamiento de enfermedades causadas por hongos dimorfos. El posaconazol no es metabolizado por enzima de la familia del citocromo P450 y es excretado principalmente en las heces. El posaconazol es un inhibidor del citocromo $\mathrm{P} 450$ 3A4, pero no inhibe la actividad de otras enzimas de la familia del citocromo $\mathrm{P} 450$. El posaconazol, por lo tanto, comparado con otros azoles, puede tener una menor cantidad de interacciones con otros medicamentos. La dosis de posaconazol es de $800 \mathrm{mg}$ al día para tratar la coccidioidomicosis generalizada (Ajithdoss et al., 2011; Welsh et al., 2012).

La caspofungina es una equinocandina que ha mostrado cierta eficacia en modelos murinos, con una mejor correlación in vitro/in vivo, utilizando una concentración mínima efectiva (CME), en lugar de una concentración mínima inhibitoria (CMI) (de Aguiar Cordeiro et al., 2009). Se recomienda suministrar dosis bajas del antimicótico después de la desaparición de los signos para evitar de esa forma casos recurrentes en algunos animales. Un antimicótico muy empleado en micosis sistémicas es la anfotericina $\mathrm{B}$, aunque para el tratamiento de la coccidioidomicosis parece no ser de mucha ayuda (Ettinger \& Feldman, 2004).

En la actualidad se están probando fármacos antifúngicos en desarrollo como el VT-1161, el cual resultó eficaz para el tratamiento de formas respiratorias en perros naturalmente infectados, con una toxicidad mínima. Este hecho promueve investigaciones para su uso en humanos (Shubitz et al., 2017).

Con respecto al seguimiento clínico, la mejor manifestación de mejora es la desaparición de los signos de la enfermedad. Cuando se realizan tratamientos con antimicóticos nitrogenados durante largos períodos se debe evaluar el perfil completo de enzimas hepáticas séricas (Ettinger \& Feldman, 2004; Morgan et al., 2004). Se deben evaluar los títulos de anticuerpos 3 y 6 meses después de los tratamientos para controlar la reactividad de la infección (Ettinger \& Feldman, 2004).

El pronóstico en perros y gatos es bueno cuando solo se presentaron manifestaciones pulmonares. En animales con enfermedad diseminada el pronóstico es reservado; los individuos con enfermedad diseminada y suspensión del tratamiento al desaparecer los signos clínicos, generalmente padecen recaídas. La enfermedad diseminada sin tratar siempre es fatal (Morgan et al., 2004). 


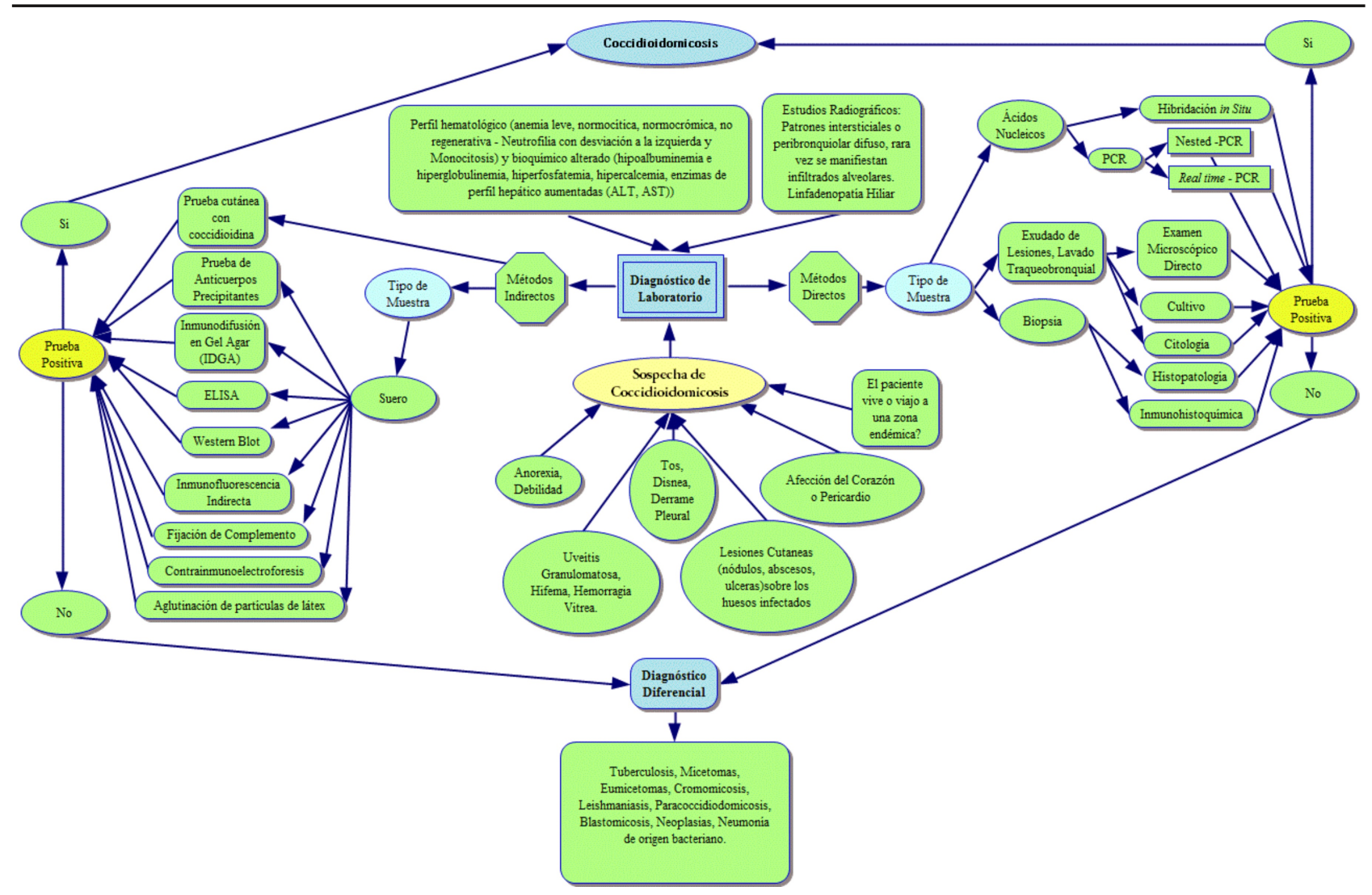

Figura 3. Algoritmo de diagnóstico de coccidioidomicosis.

\section{Caso clínico}

En Catamarca, Argentina, el propietario de un perro de raza boxer, macho, de 2 años y 10 meses de edad, tratado anteriormente con corticoides y antibióticos por un cuadro pulmonar crónico, llegó a la consulta presentando tos, disnea, hipertermia, claudicación, emaciación, lesiones plantares ulceradas y adenopatías submaxilares y poplíteas. El médico veterinario le indicó estudios radiográficos, los cuales mostraron una neumonitis con patrón intersticial, y análisis de sangre que indicaron una leucocitosis con neutrofilia e hiperglobulinemia.

El veterinario decidió obtener una biopsia de las lesiones cutáneas para su estudio histopatológico. En el mismo se observaron granulomas histiocitarios subepidérmicos y esférulas compatibles con Coccidioides sp. Además, se realizaron estudios microbiológicos de linfonódulo submaxilar y serológicos para la detección de anticuerpos anti-Coccidioides. El examen directo aclarado con $\mathrm{KOH}$, la inmunohistoquímica y otras coloraciones del tejido ganglionar revelaron presencia de esférulas y endosporas.

Se medicó al paciente con fluconazol durante los primeros 4 días de tratamiento y al quinto se decidió cambiar por itraconazol en una dosis de $100 \mathrm{mg} /$ día. El paciente no presentó mejoría y se resolvió la eutanasia, debido al avanzado e irreversible cuadro clínico.
El hongo fue identificado como Coccidioides sp. mediante cultivo y conversión dimórfica en medio de Converse y detección de antígenos extracelulares. Posteriormente, utilizando técnicas moleculares, el hongo pudo ser identificado como $C$. posadasii mediante secuenciación de un fragmento del gen Ag2/PRA (Dra. Canteros, comunicación personal) (Casal et al., 2008).

\section{Algoritmo de diagnóstico}

En la figura 3 se muestra un algoritmo simple para arribar al diagnóstico de la coccidioidomicosis. Es recomendable que se obtengan resultados positivos de tres o más pruebas para confirmar el diagnóstico.

\section{Discusión y conclusiones}

El cultivo positivo de Coccidioides spp. aun se considera el estándar de oro para el diagnóstico de esta micosis. Sin embargo, la dificultad para la obtención de un aislamiento hace que la utilización de métodos serológicos sea más apropiada (Laniado-Laborin et al., 2012).

En estudios citológicos, dependiendo del material a examinar, puede observarse una escasa cantidad de esférulas, lo que hace dificultoso el 
diagnóstico. Para llegar a un diagnóstico exitoso es necesario contar con el material clínico adecuado, en abundante cantidad, y conservarlo convenientemente hasta el momento de analizarlo. En estudios histopatológicos se recomienda tomar muestras de varios sitios para aumentar las posibilidades de encontrar el microorganismo. Las tinciones recomendadas son PAS y PMN (Morgan et al., 2004). El estudio histopatológico se considera más factible que las técnicas moleculares, debido a que existen pocos laboratorios para el diagnóstico de coccidioidomicosis mediante PCR, así como para otras micosis sistémicas (RamírezRomero et al., 2016).

En los perros que presenten una signología de debilidad crónica, linfadenopatía generalizada, cojera y nódulos o úlceras cutáneas debe considerarse, dentro de los diagnósticos diferenciales, una micosis sistémica, entre las que se encuentra la coccidioidomicosis, sobre todo en regiones en las que estas enfermedades fúngicas son consideradas endémicas. Se han realizado estudios retrospectivos para determinar la asociación de lesiones glomerulares con la coccidioidomicosis mediante histopatología, ya que un análisis de orina no es frecuente ante una sospecha de coccidioidomicosis (Mehrkens et al., 2016). Sin embargo, se requieren más investigaciones con técnicas que permitan demostrar la presencia de complejos inmunes asociados a la producción de una glomerulonefritis en estos casos.

En regiones donde no es endémica, la coccidioidomicosis representa un desafío para el diagnóstico, debido a que su presentación clínica no es específica y puede sugerir otras enfermedades, incluyendo tuberculosis (Fernández et al., 2017). Es indispensable emplear varias de las técnicas disponibles para llegar a un diagnóstico definitivo, tanto en humanos como animales.

La combinación de terbinafina y un triazol puede tener efectos sinérgicos en el tratamiento (Simões et al, 2016). No obstante, se necesitan estudios más exhaustivos para evaluar su eficiencia en la resolución de la enfermedad.

La coccidioidomicosis es una enfermedad micótica de gran relevancia a causa de la gran variedad de especies que pueden verse afectadas, incluyendo al ser humano. Al tratarse de un problema de salud pública, el control y la vigilancia de esta enfermedad deben integrar los conocimientos de los médicos veterinarios y los médicos, sobre todo en regiones en las que la presentación de esta enfermedad no es frecuente.

\section{Conflicto de intereses}

Los autores declaran que no existe conflicto de intereses, incluyendo entre estos últimos las relaciones financieras, personales o de otro tipo con otras personas $\mathrm{u}$ organizaciones que pudieran influir de manera inapropiada en el trabajo.

\section{Bibliografía}

Ajithdoss D K, Trainor KE, Snyder KD, Bridges CH, Langohr IM, Kiupel M, Porter BF. 2011. Coccidioidomycosis presenting as a heart base mass in two dogs. Journal of Comparative Pathology. 145:132-7.

doi: 10.1016/j.jcpa.2010.12.014

Binnicker MJ, Buckwalter SP, Eisberner JJ, Stewart RA, Mccullough AE, Wohlfiel SL, Wengenack NL. 2007. Detection of Coccidioides species in clinical specimens by real-time PCR. Journal of Clinical Microbiology. 45:173-8.

doi: 10.1128/JCM.01776-06

Burt A, Dechairo BM, Koenig GL, Carter DA, White TJ, Taylor JW. 1997. Molecular markers reveal differentiation among isolates of Coccidioides immitis from California, Arizona and Texas. Molecular Ecology. 6:781-6.

Calderón-Garcidueñas AL, Vázquez-Contreras JA, González-Murillo EA, Vázquez Martínez CA, CerdáFlores RM. 2013. Factores de riesgo en pacientes con coccidioidomicosis diseminada fatal. Estudio de casos y controles. Revista Española de Patología. 46:212-9.

Canteros C, Suárez-Álvarez R. 2012. Micosis Sistémicas Endémicas. (Tesina. Especialidad en Diagnóstico Veterinario de Laboratorio). Facultad de Ciencias Veterinarias. Universidad Nacional de la Plata, La Plata - Argentina.

Canteros CE, Toranzo A, Suárez-Álvarez R, Davel G, Castañón-Olivares LR, Nápoli J. 2009. Identidad genética del hongo causante del primer caso de coccidioidomicosis descripto por Alejandro Posadas en 1892. Medicina (Buenos Aires). 69:215-20.

Carrada-Bravo T. 2014. Los métodos de laboratorio en el diagnóstico de la coccidioidomicosis. Revista Chilena de Infectología. 31:293-7.

Carrasco-Zuber JE, Navarrete-Dechent C, Bonifaz A, Fich F, Vial-Letelier V, Berroeta-Mauriziano D. 2016. Afectación cutánea en las micosis profundas: una revisión de la literatura. Parte 2. Micosis sistémicas. Actas Dermo-Sifiliográficas. 107:81622.

doi: 10.1016/j.ad.2016.06.001

Casal JI, Álvarez RO, Lee W, Rivas MC, Davel G, Canteros CE. 2008. Disseminated coccidioidomycosis in a dog. Revista Argentina de Microbiología. 40:109-19.

Castañón-Olivares LR, Guereña-Elizalde D, González-Martínez MR, Licea-Navarro AF, 
González-González GM, Aroch-Calderón A. 2007. Molecular identification of Coccidioides isolates from Mexican patients. Annals of the New York Academy of Sciences. 111:326-35.

doi: $10.1196 /$ annals.1406.047

Chow NA, Lindsley MD, Mccotter OZ, Kangiser D, Wohrle RD, Clifford WR, Yaglom H D, Adams LE, Komatsu K, Durkin MM, Baker RJ, Shubitz LF, Derado G, Chiller TM, Litvintseva AP. 2017. Development of an enzyme immunoassay for detection of antibodies against Coccidioides in dogs and other mammalian species. PLoS One. 12:1-14.

doi: 10.1371/journal.pone.0175081

De Aguiar-Cordeiro R, Brilhante RS, Rocha MF, Medrano DJ, Monteiro AJ, Tavares JL, De Lima RA, De Camargo ZP, Sidrim JJ. 2009. In vitro synergistic effects of antituberculous drugs plus antifungals against Coccidioides posadasii. International Journal of Antimicrobial Agents. 34:278-80.

doi: 10.1016/j.ijantimicag.2009.04.009

DiCaudo DJ. 2006. Coccidioidomycosis: a review and update. Journal of the American Academy of Dermatology. 55:929-42.

doi: 10.1016/j.jaad.2006.04.039

Espinoza Pérez J, Agüero Balbín R, Martínez Meñaca A, Ciorba C, Mora Cuesta V. 2014. Micosis pulmonares. Medicine.11(66):3949-62.

doi: 10.1016/So304-5412(14)70867-4

Ettinger SJ, Feldman EC. 2004. Veterinary Internal Medicine. Philadelphia, Elsevier. pp. 1359-6o.

Fernández R, Arenas R, Duarte-Escalante E, FríasDe León MG, Vega Memige ME, Acosta Altamirano G, Reyes-Montes MDR. 2017. Diagnosis of coccidioidomycosis in a non-endemic area: inference of the probable geographic area of an infection. Revista Iberoamericana de Micología. 34:237-40.

doi: 10.1016/j.riam.2017.03.006

Fisher MC, Koenig GL, White TJ, Taylor J W. 2002. Molecular and phenotypic description of Coccidioides posadasii sp. nov., previously recognized as the non-California population of Coccidioides immitis. Mycologia. 94:73-84.

Gómez N, Guida N. Coccidioidomicosis. En: Gómez N, Guida N. 2010. Enfermedades infecciosas de los caninos y felinos, Ciudad Autónoma de Buenos Aires, Inter-Médica, 65-66.

Jinks MR, English RV, Gilger BC. 2016. Causes of endogenous uveitis in cats presented to referral clinics in North Carolina. Veterinary
Ophthalmology. 19(1):30-7.

doi: 10.1111/vop.12324

Koufopanou V, Burt A, Taylor JW. 1997. Concordance of gene genealogies reveals reproductive isolation in the pathogenic fungus Coccidioides immitis. Proceedings of the National Academy of Sciences. 94:5478-82.

Laniado-Laborin R, Alcantar-Schramm JM, Cazares-Adame R. 2012. Coccidioidomycosis: An update. Current Fungal Infection Reports. 6:11320.

Lloret A, Hartmann K, Pennisi MG, Ferrer L, Addie D, Belak S, Boucraut-Baralon C, Egberink H, Frymus T, Gruffydd-Jones T, Hosie MJ, Lutz H, Marsilio F, Mostl K, Radford AD, Thiry E, Truyen U, Horzinek MC. 2013. Rare systemic mycoses in cats: blastomycosis, histoplasmosis and coccidioidomycosis: ABCD guidelines on prevention and management. Journal of Feline Medicine and Surgery. 15:624-7.

doi: 10.1177/1098612X13489226

Martínez-Méndez DK, Semprún-Hernández N, Hernández-Valles RC. 2015. Coccidioidomicosis: estado actual de la endemia en Venezuela. Investigación Clínica. 56:411-20.

Mehrkens LR, Mohr FC, Sykes JE. 2016. Clinicopathologic and histopathologic renal abnormalities in dogs with coccidioidomycosis. Journal of Veterinary Internal Medicine. 30:166771.

doi: 10.1111/jvim.14573

Morgan RV, Bright R M, Swartout MS. 2004. Clínica de pequeños animales $4^{\circ}$ ed. Madrid, SA Elsevier España.

Negroni R. 2011. Historia del descubrimiento de la coccidioidomicosis. Revista Argentina de Dermatología. 92:1-15.

Ortiz-Encinas V, Lares-Villa F, Luna-Nevárez P, Reyna-Granados J, Munguía-Xóchihua J, IbarraGámez J. 2015. Estudios anatómico patológicos, serológicos e histopatológicos de perros positivos a coccidioidomicosis infectados naturalmente. Abanico Veterinario. 5:13-25.

Peng T, Orsborn KI, Orbach MJ, Galgiani JN. 1999. Proline-rich vaccine candidate antigen of Coccidioides immitis: conservation among isolates and differential expression with spherule maturation. Journal of Infectious Diseases. 179:518-21.

doi: $10.1086 / 314604$ 
Ramírez-Romero R, Silva-Pérez RA, Lara-Arias J, Ramírez-Hernández C, Marino-Martínez IA, Barbosa-Quintana A, López-Mayagoitia A. 2016. Coccidioidomycosis in biopsies with presumptive diagnosis of malignancy in dogs: report of three cases and comparative discussion of published reports. Mycopathologia. 181:151-7.

doi: 10.1007/s11046-015-9948-4

Schaer M. 2006. Medicina clínica del perro y el gato. Madrid, Elsevier España.

Shubitz LF. 2007. Comparative aspects of coccidioidomycosis in animals and humans. Annals of the New York Academy of Sciences. 1111:395-403.

doi: 10.1196/annals.1406.007

Shubitz LF, Dial SM. 2005. Coccidioidomycosis: a diagnostic challenge. Clinical Techniques in Small Animal Practice. 20:220-6.

doi: 10.1053/j.ctsap.2005.07.002

Shubitz LF, Dial SM, Galgiani JN. 2011. Tlymphocyte predominance in lesions of canine coccidioidomycosis. Veterinary Pathology. 48:1008-11.

doi: 10.1177/0300985810384410

Shubitz LF, Roy ME, Trinh HT, Hoekstra WJ, Schotzinger RJ, Garvey EP. 2017. Efficacy of the investigational antifungal VT-1161 in treating naturally occurring coccidioidomycosis in dogs. Antimicrobial Agents and Chemotherapy. 61(5): 111-7.

doi: 10.1128/AAC.00111-17

Simoes DM, Dial SM, Coyner KS, Schick AE, Lewis TP. 2nd. 2016. Retrospective analysis of cutaneous lesions in 23 canine and 17 feline cases of coccidiodomycosis seen in Arizona, USA (20092015). Veterinary Dermatology. 27:346-87.

doi: 10.1111/vde.12356

Tintelnot K, De Hoog GS, Antweile E, Losert H, Seibold M, Brandt MA, Van Den Ende A. H, Fisher MC. 2007. Taxonomic and diagnostic markers for identification of Coccidioides immitis and Coccidioides posadasii. Medical Mycology.45:38593.

doi: $10.1080 / 13693780701288070$

Umeyama T, Sano A, Kamei K, Niimi M, Nishimura K, Uehara Y. 2006. Novel approach to designing primers for identification and distinction of the human pathogenic fungi Coccidioides immitis and Coccidioides posadasii by PCR amplification. Journal of Clinical Microbiology. 44:1859-62.

doi: 10.1128/JCM.44.5.1859-1862.2006

Vale-Echeto O, Alvarado-Morillo M, FernándezOrozco E, Camacho-Bracho J, Delgado C. 1999. Coccidioidomicosis sistémica en canino: estudio radiológico y anatomopatológico de un caso. Revista Científica FCV-LUZ. 9: 267-75.

Welsh O, Vera-Cabrera L, Rendon A, González G, Bonifaz A. 2012. Coccidioidomycosis. Clinics in Dermatology. 30:573-91.

doi: 10.1016/j.clindermatol.2012.01.003

Zimmermann CR, Snedker CJ, Pappagianis D. 1994. Characterization of Coccidioides immitis isolates by restriction fragment length polymorphisms. Journal of Clinical Microbiology. 32:3040-2. 